\title{
Photoemission core level binding energies from multiple sized nanoparticles on the same support: $\mathrm{TiO}_{2}(110) / \mathrm{Au} \odot$
}

Cite as: J. Chem. Phys. 152, 024709 (2020); https://doi.org/10.1063/1.5135760

Submitted: 08 November 2019 . Accepted: 12 December 2019. Published Online: 14 January 2020

Andrew Mellor, Axel Wilson (D), Chi L. Pang (D), Chi M. Yim (D), Francesco Maccherozzi, Sarnjeet S. Dhesi, Christopher A. Muryn, Hicham Idriss, and Geoff Thornton (D)

\section{COLLECTIONS}

Note: This article is part of the JCP Special Topic on Oxide Chemistry and Catalysis.

F This paper was selected as Featured
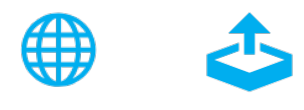

\section{ARTICLES YOU MAY BE INTERESTED IN}

Analytical classical density functionals from an equation learning network

The Journal of Chemical Physics 152, 021102 (2020); https://doi.org/10.1063/1.5135919

Transient absorption microscopy: Technological innovations and applications in materials science and life science

The Journal of Chemical Physics 152, 020901 (2020); https://doi.org/10.1063/1.5129123

Assessment of dynamic structural instabilities across 24 cubic inorganic halide perovskites The Journal of Chemical Physics 152, 024703 (2020); https://doi.org/10.1063/1.5131575

\section{Lock-in Amplifiers up to $600 \mathrm{MHz}$}
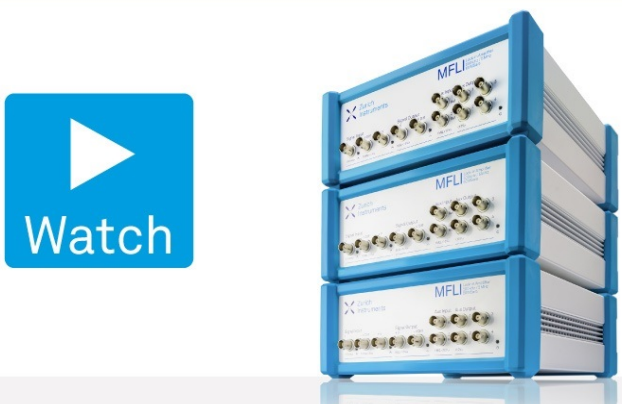


\title{
Photoemission core level binding energies from multiple sized nanoparticles on the same support: $\mathrm{TiO}_{2}(110) / \mathrm{Au}$
}

\author{
Cite as: J. Chem. Phys. 152, 024709 (2020); doi: 10.1063/1.5135760 \\ Submitted: 8 November 2019 - Accepted: 12 December 2019 • \\ Published Online: 14 January 2020
}

Andrew Mellor, ${ }^{1}$ Axel Wilson, ${ }^{2}$ (D) Chi L. Pang, ${ }^{1}$ (D) Chi M. Yim, ${ }^{1}$ (D) Francesco Maccherozzi, ${ }^{2}$

Sarnjeet S. Dhesi, ${ }^{2}$ Christopher A. Muryn, ${ }^{3}$ Hicham Idriss, ${ }^{1,4}$ and Geoff Thornton ${ }^{1, a)}$ (D)

\begin{abstract}
AFFILIATIONS
'Department of Chemistry and London Centre for Nanotechnology, University College London, 20 Gordon Street, London WCIH OAJ, United Kingdom

²Diamond Light Source, Harwell Science and Innovation Campus, Oxfordshire OX11 ODE, United Kingdom

${ }^{3}$ School of Chemistry and Photon Science Institute, The University of Manchester, Oxford Road, Manchester M13 9PL, United Kingdom

4SABIC - CRI, KAUST, Jeddah 23955, Saudi Arabia
\end{abstract}

Note: This article is part of the JCP Special Topic on Oxide Chemistry and Catalysis.

${ }^{a)}$ Author to whom correspondence should be addressed: g.thornton@ucl.ac.uk

\begin{abstract}
A novel method of measuring the core level binding energies of multiple sized nanoparticles on the same substrate is demonstrated using the early stage of $\mathrm{Au}$ nanoparticle growth on reduced $\mathrm{r}-\mathrm{TiO}_{2}(110)$. This method employed in situ scanning tunneling microscopy (STM) and microfocused X-ray photoemission spectroscopy. An STM tip-shadowing method was used to synthesize patterned areas of Au nanoparticles on the substrate with different coverages and sizes. Patterns were identified and imaged using a UV photoelectron emission microscope. The $\mathrm{Au} 4 \mathrm{f}$ core level binding energies of the nanoparticles were investigated as a function of Au nanoparticle coverage and size. A combination of initial and final state effects modifies the binding energies of the Au $4 \mathrm{f}$ core levels as the nanoparticle size changes. When single Au atoms and $\mathrm{Au}_{3}$ clusters are present, the $\mathrm{Au} 4 \mathrm{f}_{7 / 2}$ binding energy, $84.42 \mathrm{eV}$, is similar to that observed at a high coverage (1.8 monolayer equivalent), resulting from a cancellation of initial and final state effects. As the coverage is increased, there is a decrease in binding energy, which then increases at a higher coverage to $84.39 \mathrm{eV}$. These results are consistent with a Volmer-Weber nucleation-growth model of Au nanoparticles at oxygen vacancies, resulting in electron transfer to the nanoparticles.
\end{abstract}

Published under license by AIP Publishing. https://doi.org/10.1063/1.5135760

\section{INTRODUCTION}

Photoelectron spectroscopy (PES) has been widely employed to explore the electronic characteristics of nanoparticles and their interactions with single crystalline substrates. ${ }^{1-3}$ Here, we refer to earlier studies of nanoparticles on $\mathrm{TiO}_{2}(110)^{1-3}$ since this is the model system studied in the present work. Au nanoparticles are chosen because of their significance in several industrial processes, including oxidation and water gas shift catalysis. ${ }^{4}$ The choice of support material has been shown to have a critical effect on the catalytic activity of nanoparticles, with $\mathrm{TiO}_{2}$ being particularly effective.
Moreover, the question of charge transfer between the nanoparticle and the substrate is a much-debated aspect of Au based catalysis. ${ }^{6,9-11}$ The surface chemistry of $\mathrm{Au}$ supported on $\mathrm{TiO}_{2}$ has been recently reviewed. ${ }^{12}$

PES core level electron binding energy shifts of nanoparticles can be explained by changes in electron distributions and, in some cases, the charge transfer between a nanoparticle and the substrate. ${ }^{1,2,13,14}$ In the case of $\mathrm{Au}$ nanoparticles, the binding energy of core level electrons has been shown to vary with the nanoparticle size on polycrystalline $\mathrm{TiO}_{2} .{ }^{13}$ In addition to the $\mathrm{Au}$ charge state and particle size, the extent to which the substrate has been reduced prior 
to $\mathrm{Au}$ deposition has been shown to influence the catalytic activity of the supported nanoparticles ${ }^{12,15}$ and the degree of charge transfer. ${ }^{12,14,15}$ Therefore, describing the interactions between the centers of reduction (e.g., oxygen vacancies) and the Au nanoparticles is key to understanding the relationship between the catalytic activity and the reduction state of the substrate. There are many independent studies that have examined the nature of electron transfer occurring between $\mathrm{Au}$ nanoparticles and reduced $\mathrm{TiO}_{2}$. The consensus has been that anionic gold is formed at a low coverage in UHV, although recent calculations suggest that neutral or cationic gold is obtained. ${ }^{1}$

Reduction of $\mathrm{TiO}_{2}(110)$ gives rise to an electronic state within the bandgap of the material. ${ }^{17}$ The presence of this band-gap-state (BGS) is associated with the generation of defects such as interstitial $\mathrm{Ti}$ atoms and bridging oxygen vacancies (Ov) in the bulk and at the surface, respectively. ${ }^{18-20}$ Oxygen vacancies on rutile $\mathrm{TiO}_{2}(110)$ act as electron trap sites and have been shown to play a role in surface-mediated molecular reactions. ${ }^{21}$ They are associated with an area of excess electron density, ${ }^{21-23}$ and low temperature scanning tunneling microscopy (STM) has been used to show that this has polaronic character. ${ }^{21,24}$ Previous experimental and theoretical work has pointed to the initial adsorption of $\mathrm{Au}$ atoms, $\mathrm{Au}_{1}$, at $\mathrm{Ov}$ sites on reduced $\mathrm{r}-\mathrm{TiO}_{2}(110){ }^{15,25-27}$ This has recently been directly visualized using STM. ${ }^{28} \mathrm{Au}_{1}$ and $\mathrm{Au}_{3}$ are bound at $\mathrm{Ov}$, but larger nanoparticles are mobile at room temperature and agglomerate to form larger particles with an increased coverage.

Interpretation of core level electron binding energy shifts of nanoparticles as a function of size is made difficult by the presence of both initial and final state effects. ${ }^{3,14}$ Initial state effects arise from intrinsic properties of the nanoparticles, i.e., the initial electronic state of the system. For metal nanoparticles on a metal oxide, this would be evident if there was charge transfer. For example, if the nanoparticle becomes positively charged, then the binding energy will increase. Final state effects arise from the photoemission process, i.e., its electronic state immediately after photoemission. Final state effects diminish as the particle size increases due to the screening of the newly generated core hole by its local electronic environment. Hence, the final state binding energy shift becomes less positive as the particle size increases. For $\mathrm{Au}$ on stoichiometric $\mathrm{TiO}_{2}(110)$, earlier work observed a decrease in $\mathrm{Au}$ 4f binding energies with an increase in Au coverage. In contrast, for reduced $\mathrm{TiO}_{2}(110)$, the binding energy initially decreased but then increased at a higher coverage. The latter behavior is ascribed to a reduction in the averaged charge transfer to the nanoparticles at higher coverages. $^{14}$

A potential drawback in studies of reducible oxides such as $\mathrm{TiO}_{2}$ is that different levels of reduction can be inadvertently introduced during a series of measurements, which can modify the degree of charge transfer to/from supported nanoparticles. In this work, we demonstrate a novel experimental approach employing microfocused X-ray photoemission spectroscopy ( $\mu$-XPS) and STM that can be employed to study multiple sized nanoparticles on the same substrate. Au nanoparticles were deposited onto $\mathrm{TiO}_{2}(110)$ in discrete regions using a scanning tunneling microscope tip shadowing effect, with each region containing Au nanoparticles of a different size distribution. This minimizes any discrepancies arising from the variable reduction state of the substrate, such as defect density. Moreover, imaging the nanoparticles in situ removes any uncertainty regarding the size of the nanoparticles and their binding sites.

\section{EXPERIMENTAL AND INSTRUMENTAL DETAILS}

The experiment was carried out on the XPEEM end-station of the I06 Nanoscience beamline at Diamond Light Source. This end station has a preparation chamber equipped with STM (Omicron VT STM), low energy electron diffraction (LEED)/retarding field Auger electron spectroscopy (AES), and an analysis chamber equipped with an X-ray photoelectron microscopy (XPEEM) instrument (Elmitec SPELEEM-III), which can also be used to image with low energy electron microscopy (LEEM) and UV-PEEM with a $\mathrm{Hg}$ lamp (Quantum Design Europe). Both chambers operate at a base pressure in the $10^{-10}$ mbar region. $\mu$-XPS spectra were acquired in dispersive mode at a photon energy of $200 \mathrm{eV}$ with a field of view of $8 \mu \mathrm{m}$. The photoelectron energy was calibrated using the Fermi level and the bandgap state. This was systematically cross-checked using the Ti $3 p$ binding energy.

Two $\mathrm{TiO}_{2}(110)$ ( $\left.\mathrm{MaTecK} \mathrm{GmbH}\right)$ samples were introduced into the UHV system. Both were prepared by five cycles of $1 \mathrm{keV} \mathrm{Ar}+$ sputtering and annealing at $1100 \mathrm{~K}$ for $10 \mathrm{~min}$. After preparation, a sharp $(1 \times 1)$ LEED pattern was observed and the absence of surface contamination was confirmed using AES. The clean samples were then introduced into the STM, where images of the clean surface were recorded to check the presence of large terraces. Au deposition was carried out at room temperature using an e-beam evaporator (Omicron EFM3) oriented toward the STM tip in its scanning configuration. The evaporator built-in flux monitor was stabilized for a given flux, which was constant throughout the experiment. The height histogram of a large assembly of nanoparticles, typically over 100, was obtained using STM. For $\mathrm{Au}_{1}$ and $\mathrm{Au}_{3}$, the nanoparticles were simply counted. The monolayer equivalent (MLE) coverage was then determined by summing all the heights with the assumption that the nanoparticles for $\mathrm{Au}_{4}$ and larger are hemispherical and that the lattice parameter of $\mathrm{Au}$ in nanoparticles is that of the bulk, $4.08 \AA .^{30} 1$ MLE corresponds to the atomic density of one layer of $\mathrm{Au}\langle 111\rangle: 1.39 \times 10^{15}$ at. $\mathrm{cm}^{-2}$.

To produce a stepped gradient of the $\mathrm{Au}$ nanoparticle size over the surface, a STM tip shadowing effect was employed. ${ }^{31,32}$ In this methodology, the STM tip acts as a shutter, blocking the pathway between an area of the sample and the incoming metal atoms emitted by a line of sight evaporator. By moving the STM tip over the surface in the precise direction of the evaporator, the previously shadowed area of the surface is progressively exposed to the incoming flux of metal atoms. This gives rise to a continuously varying gradient of nanoparticle coverage on the surface of the substrate. By halting metal deposition during each incremental movement of the STM tip (i.e., closing the shutter on the evaporator; see Fig. 1), well-defined discrete areas of monotonic nanoparticle coverage could be deposited, rather than a continuously varying gradient.

The sample was then transferred to the XPEEM analysis chamber, where a few-micrometers wide patterns created using STM were identified using UV-PEEM. This imaging method offers the best contrast between areas of Au nanoparticles that have different but similar diameters. As the tip shadow area measures only $100 \mathrm{~nm}$ 


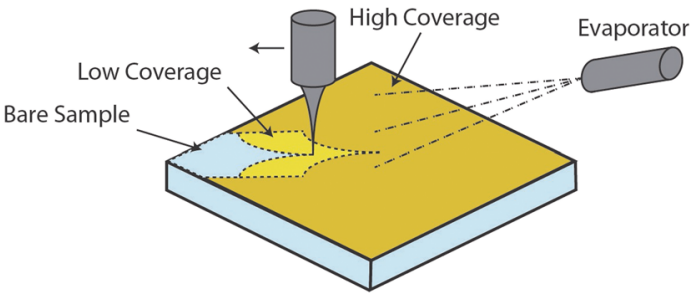

FIG. 1. Schematic diagram of the STM-tip shadow method used to deposit discrete regions of different Au coverages onto a $\mathrm{TiO}_{2}$ substrate. The motion of the tip between deposition stages is indicated in the diagram. Two tip-shadows are shown: the first (outer) exhibits a lower Au coverage than the nonshadowed area of the sample while the second (inner) shows the bare substrate.

at its apex (see below), it was not feasible to locate it by exploring the entire sample with UV-PEEM. To circumvent this problem, we employed an area retrieval method detailed in the next paragraph. This was also employed to optimize the tilt of the sample, which is an important parameter during the PEEM alignment. It effectively defines an area of the sample where lateral movement using the in-plane piezomotor translations does not compromise the alignment of the sample and the analyzer. This allows for lateral movement, within a range of approximately $500 \mu \mathrm{m}$, from one area of interest to another with no impact on the reliability of the $\mu$-XPS signal.

The definition of a set of coordinates that can be located and recalled in both the STM and XPEEM represents a critical step in this experiment. To do so, a special sample (Siemens $\operatorname{Star}^{T M}$, shown in Fig. 2) was used. This has a diameter of $500 \mu \mathrm{m}$ with a $5 \mu \mathrm{m}$ ring that marks its exact centre, which is visible both optically, via the STM CCD camera, and with LEEM. The coordinates of the tilt free zone were then indexed using LEEM, and the optimal area for depositing the nanoparticle shadow pattern was determined. The star sample was then transferred to the STM, which was equipped with a CCD camera capable of resolving movements of the STM tip as small as $30 \mu \mathrm{m}$ over a sample's surface. LEEM relative coordinates of the STM

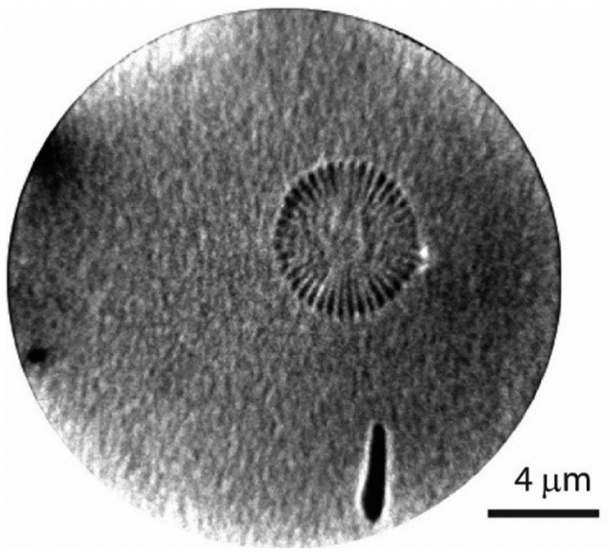

FIG. 2. LEEM image taken from the Siemens $\operatorname{Star}^{\top \mathrm{M}}$ sample showing the central ring feature. Electron energy $=-0.34 \mathrm{eV}$ and $\mathrm{FOV}=20 \mu \mathrm{m}$.

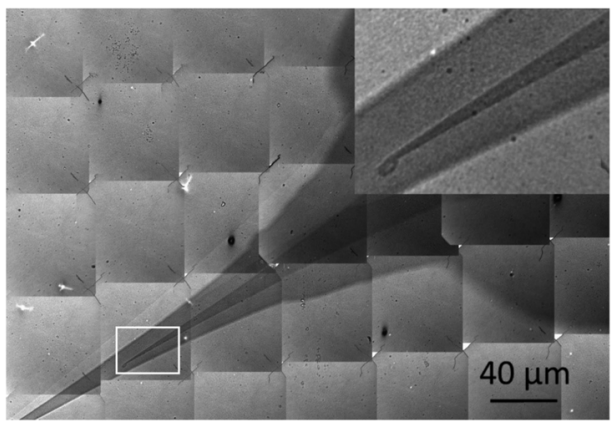

FIG. 3. A series of stitched UV-PEEM images taken from $\mathrm{TiO}_{2}(110)$ after the deposition of $0.4 \mathrm{MLE}$ Au showing a tip shadow extending diagonally down from right to left as an area of darker contrast corresponding to lower coverages $(0.3$ and $0.05 \mathrm{MLE})$. Within the first "outer" shadow, a second "inner" shadow can be seen. The inset image shows a magnified view of the apex of the inner tip shadow. The inner shadow was well enough resolved to leave an impression of the "loop" at the end of the tip. Image contrast is derived from the local work function of the sample. Electron energy $=0.5 \mathrm{eV}$ and FOV $=80 \mu \mathrm{m}$ per image taken.

tip were determined and recorded with respect to the optically visible central ring. For future samples, the optimum position of the STM tip for nanoparticle shadowing (i.e., within the tilt free zone) could be recalled. This position was then located using the STM coordinate system before recording an image. After creating the tipshadowed $\mathrm{Au} / \mathrm{TiO}_{2}$ sample and having defined the position of the shadow's apex in both the STM and XPEEM apparatus, the sample's surface was imaged using UV-PEEM. Figure 3 shows a series of stitched UV-PEEM images taken from the $\mathrm{Au} / \mathrm{TiO}_{2}$ sample. In the images, multiple well-defined impressions of the STM tip are seen on the surface, which become incrementally darker toward the sample's edge. The edge of each STM tip impression represents the boundary between two Au coverage regions. The change in image contrast can be explained by an increased local work function of the surface for areas with a higher Au coverage. ${ }^{33}$ The inset of Fig. 3 shows a magnified view of the apex of an STM tip impression (nanoparticle shadow). This is also evident in an STM image of an area of the shadowed surface (see Fig. 4).

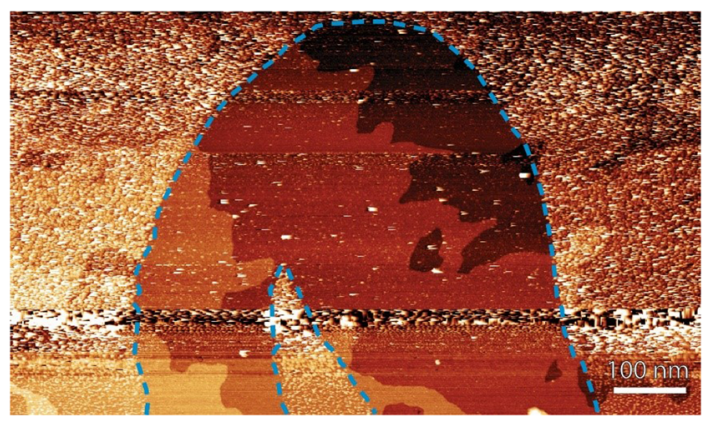

FIG. 4. $1000 \times 575 \mathrm{~nm}^{2}$ STM image $(2 \mathrm{~V}, 100 \mathrm{pA})$ of $\mathrm{TiO}_{2}(110)$ after the deposition of $0.4 \mathrm{MLE}$ Au using the tip-shadow method. The blue dashed line indicates the edge of the tip-shadow. 


\section{RESULTS AND DISCUSSION}

A feature of $\mu$-XPS when used in the study of reducible metal oxides is that the high brightness of the incident X-rays can induce beam damage in the form of reduction. This has been observed for $\mathrm{TiO}_{2}{ }^{34,35}$ and $\mathrm{CeO}_{2} .{ }^{36}$ This effect is likely caused by ionization, excitation, and de-excitation events that reduce the surface. ${ }^{37,38}$ For $\mathrm{CeO}_{2}(111)$, this effect was removed by measuring in a partial pressure of $\mathrm{O}_{2} \cdot{ }^{36}$ We adopt this methodology in the present work, its efficacy being demonstrated by the $\mu$-XPS results shown in Fig. 5 . In the presence of $10^{-6} \mathrm{mbar}_{2}$, there is no X-ray induced reduction of Au-nanoparticle decorated $\mathrm{TiO}_{2}(110)$, as evidenced by the absence of an increase in the bandgap state peak at $0.8 \mathrm{eV}$ BE and $\mathrm{Ti} 3 \mathrm{p}$ features in the range $34-36 \mathrm{eV} \mathrm{BE}$. Reducing the pressure of $\mathrm{O}_{2}$ results in the appearance of these features within a few seconds, indicating reduction to $\mathrm{Ti}^{3+}$ and $\mathrm{Ti}^{2+}{ }^{19}$ There is a corresponding increase in the intensity of bandgap states that arise from Ti $3 \mathrm{~d}$ electrons. Subsequently, an attempt to reoxidize the sample was made by reintroducing $1 \times 10^{-6}$ mbar of $\mathrm{O}_{2}$ into the chamber, although this did not result in a complete reoxidation.

To exclude the possibility that Au nanoparticles on $\mathrm{TiO}_{2}(110)$ are modified by the X-ray beam, the Au $4 \mathrm{f}$ signal from a 0.4 MLE covered surface was measured as a function of $\mathrm{O}_{2}$ partial pressure. The evolution of the Au 4f $f_{5 / 2,7 / 2}$ spectra shown in Fig. 6 was recorded immediately after the acquisition of the corresponding $\mathrm{Ti} 3 \mathrm{p}$ and bandgap spectra presented in Fig. 5. The spectra in Fig. 6 show that

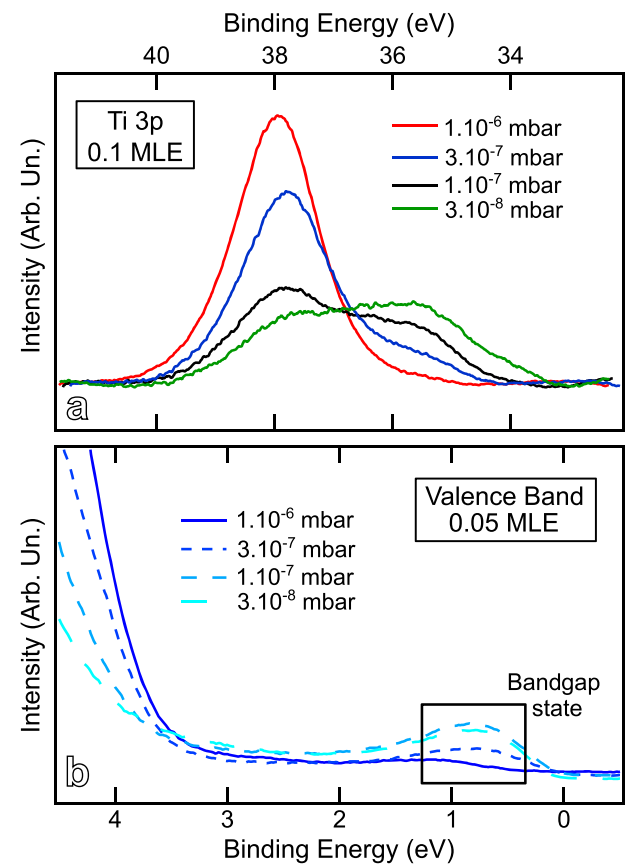

FIG. 5. (a) Ti 3p XPS spectra ( $h v=200 \mathrm{eV}$ ) obtained on a sample obtained by depositing $0.1 \mathrm{MLE}$ of Au at room temperature onto $\mathrm{TiO}_{2}(110)$. The solid lines represent spectra collected at an incrementally lower $\mathrm{O}_{2}$ partial pressure. (b) Bandgap region XPS spectra $(h v=200 \mathrm{eV})$ recorded as the $\mathrm{O}_{2}$ partial pressure decreased. The $\mathrm{TiO}_{2}$ bandgap states at $0.8 \mathrm{eV}$ below the Fermi energy are indicated.

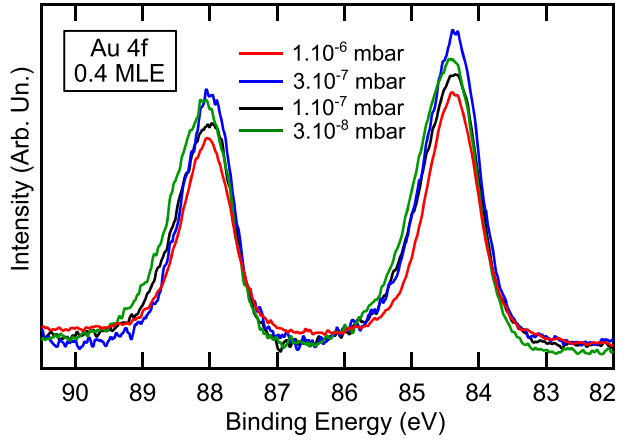

FIG. 6. Au 4f XPS spectra $(h v=200 \mathrm{eV})$ obtained on a sample composed of $0.40 \mathrm{MLE}$ of $\mathrm{Au}$ deposited at room temperature onto $\mathrm{TiO}_{2}(110)$. Spectra were recorded as a function of $\mathrm{O}_{2}$ partial pressure.

the peak intensities are not affected by the decrease in oxygen partial pressure. The slight shift in energy observed at $3 \times 10^{-8} \mathrm{mbar}$ of $\mathrm{O}_{2}$ can tentatively be attributed to the interaction between $\mathrm{Au}$ NPs and highly reduced Ti species. This last point means that the interpretation of spectrum acquired at low $\mathrm{O}_{2}$ partial pressure has to consider beam-induced reduction of the substrate. Here, we focus on $\mathrm{Au} 4 \mathrm{f}$ data collected at $10^{-6} \mathrm{mbar}$ of $\mathrm{O}_{2}$, where the chemical shift is independent of beam exposure.

We now turn to the variation of the electronic structure of $\mathrm{Au}$ nanoparticles as a function of size. This was monitored through the $\mathrm{Au} 4 \mathrm{f}$ binding energy shift for six different coverages obtained on two $\mathrm{TiO}_{2}(110)$ single crystal samples, one of them reprepared once. The first sample supported a uniform coverage of 1.8 MLE of $\mathrm{Au}$. The second and third samples were prepared using STM tipshadowing to support coverages of $0.4,0.1$, and 0.05 MLE, and 0.2 and 0.3 MLE, respectively. As noted above, this method of nanoparticle growth is employed to minimize effects arising from a variation in the level of substrate reduction.

The coverages at which Au $4 \mathrm{f}$ spectra were obtained are calculated from the analysis of the STM images shown in Fig. 7, which cover the Au coverage range 0.007-1.8 MLE. For 1.8 MLE coverage, the $\mathrm{Au} 4 \mathrm{f}$ spectrum (see Fig. 8) corresponds to the surface imaged by STM [Fig. 7(f)]. To obtain the remaining Au 4 f coverages, we employed the STM images to calibrate the Au doser. At the lowest coverage, the image contains only $\mathrm{Au}_{1}$ and $\mathrm{Au}_{3}$ nanoparticles, identified by their height of $1.5 \AA$ and $3 \AA$, respectively. ${ }^{15}$ At higher coverages, these species are still present, but larger nanoparticles increasingly dominate. As an example of the height distribution observed, the histograms measured from the images in Figs. 7(c) and 7(f) are shown in Fig. 8.

The $\mathrm{Au} 4 \mathrm{f}$ spectra were recorded for six coverages $(0.05,0.1$, $0.2,0.3,0.4,0.5$, and $1.8 \mathrm{MLE})$ at four different partial pressures of oxygen $\left(1 \times 10^{-6}, 3 \times 10^{-7}, 1 \times 10^{-7}\right.$, and $\left.3 \times 10^{-8} \mathrm{mbar}\right)$. For a given coverage, the binding energy and intensity of the Au $4 \mathrm{f}_{5 / 2,7 / 2}$ components are independent of the oxygen partial pressure. This indicates that any beam damage occurring on the substrate in the vicinity of the nanoparticles does not affect the Au $4 \mathrm{f}$ binding energies. The data recorded at an oxygen partial pressure of $10^{-6}$ mbar are shown in Fig. 9 with the Au $4 f_{7 / 2}$ binding energy variation with the coverage 

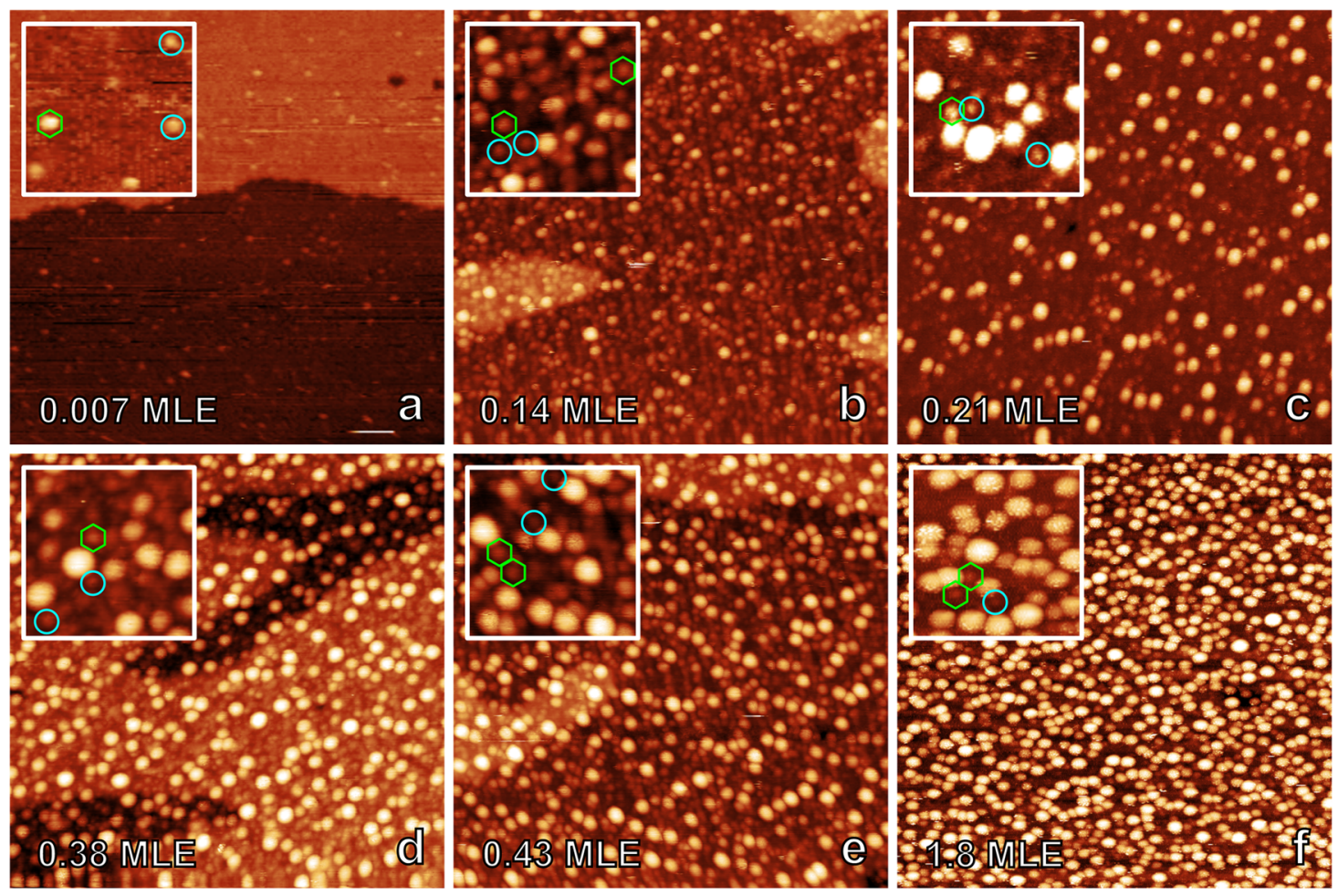

FIG. $7.50 \times 50 \mathrm{~nm}^{2}$ STM images (inset $10 \times 10 \mathrm{~nm}^{2}$ ) obtained at room temperature after the deposition of Au: $0.007,0.14,0.21,0.38,0.43$, and $1.8 \mathrm{MLE}$ for the images (a)-(f), respectively. In the inset, the green hexagons correspond to $\mathrm{Au}_{3}$ clusters and cyan circles to $\mathrm{Au}_{1}$ clusters.

plotted in Fig. 10. They display a clear binding energy shift that can be separated into two regions. In the first region, corresponding to 0.05 to $0.3 \mathrm{MLE} \mathrm{Au}$, the $\mathrm{Au} 4 \mathrm{f}_{7 / 2}$ peak shifts by $-109 \mathrm{meV}$. In the second region, corresponding to 0.3 to $1.8 \mathrm{MLE} \mathrm{Au}$, the $\mathrm{Au} 4 \mathrm{f}_{7 / 2}$ peak shifts by $+80 \mathrm{meV}$ (from 84.31 to $84.39 \mathrm{eV}$ ).

As noted above, studies of stoichiometric surfaces report only a negative binding energy shift of the $\mathrm{Au} 4 \mathrm{f}$ signal as Au coverage increases. This was ascribed to a final state effect. In contrast, for a reduced surface, an initial decrease in binding energy is followed

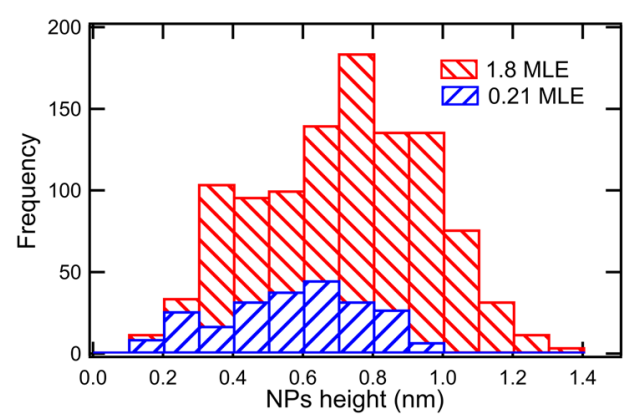

FIG. 8. Au nanoparticle height histograms measured from the STM data in Figs. 7(c) and 7 (f). The heights were obtained by measuring line profiles over individual nanoparticles. by an increase in binding energy at a higher coverage, arising from a reduction in the charge transfer per $\mathrm{Au}$ atom. ${ }^{14}$ The same trend is seen in the present work, although details of the binding energies differ, presumably because of differences in the deposition conditions that give rise to different particle sizes in the earlier work.

In addition to measuring the binding energy shifts on substrates with the same level of surface reduction, we have access to the nanoparticle size distribution through our in situ STM measurements. This allows additional qualitative comments on the

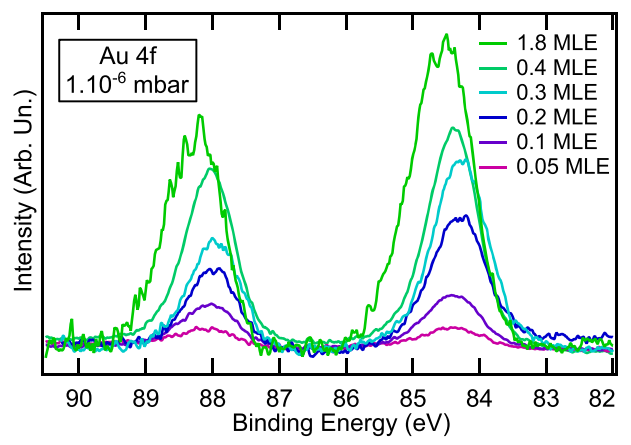

FIG. 9. Au $4 \mathrm{f} \mu$-XPS spectra $(\mathrm{h} v=200 \mathrm{eV})$ of six Au coverages of nanoparticles on $\mathrm{TiO}_{2}(110)$. All measurements were carried out in an oxygen partial pressure of $10^{-6}$ mbar. 


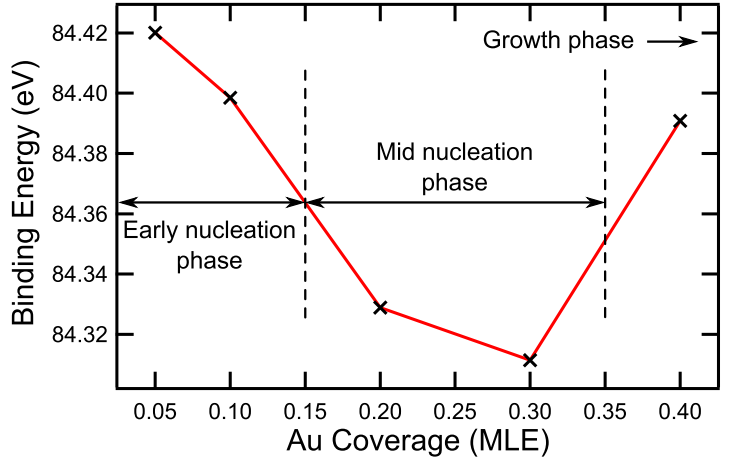

FIG. 10. Au $4 f_{7 / 2}$ binding energy as a function of nanoparticle coverage, obtained from the spectra in Fig. 9. The binding energy corresponding to $1.8 \mathrm{MLE}$ is $84.39 \mathrm{eV}$.

binding energy shifts to be made. The predominant species observed below $0.05 \mathrm{MLE}$ are $\mathrm{Au}_{1}$ and $\mathrm{Au}_{3}$. The reason is that these species grow rather than larger particles pointing to the reduced nature of the substrate, which contains oxygen vacancies. Hydroxylated surfaces are known to form larger nanoparticles. ${ }^{15}$ Both $\mathrm{Au}_{1}$ and $\mathrm{Au}_{3}$ are bound to oxygen vacancies on the basis of earlier work, ${ }^{15,28}$ which is expected to give rise to charge transfer to the nanoparticles to form negatively charged $\mathrm{Au} .{ }^{14}$ This should shift the $\mathrm{Au} 4 \mathrm{f}_{7 / 2}$ peak to lower binding energy, although the lack of screening of the core hole in such small, well-dispersed nanoparticles will provide a shift in the opposite direction. These effects essentially balance each other, resulting in an $\mathrm{Au} 4 \mathrm{f}_{7 / 2}$ binding energy of $84.42 \mathrm{eV}$ similar to that at $1.8 \mathrm{MLE}$ of $84.39 \mathrm{eV}$. At higher coverages, the size distribution increases substantially (see Fig. 8) with $\mathrm{Au}_{1}$ and $\mathrm{Au}_{3}$ still visible in the 1.8 MLE-coverage STM image [Fig. 7(f)]. This mid-nucleation phase arises when a significant proportion of the surface Ov has been filled, and a subsequent addition of Au results in diffusion and attachment of atoms to an existing nanoparticle.

An earlier room temperature STM study found the transition between nucleation and growth of $\mathrm{Au}$ on $\mathrm{TiO}_{2}(110)$ to occur around 0.15-0.2 MLE, ${ }^{39}$ consistent with the results presented here. The corresponding shift of $\mathrm{Au} 4 \mathrm{f}_{7 / 2}$ to lower binding energy with an increase in the mean nanoparticle diameter (Figs. 9 and 10) indicates that a reduction in the final state effect dominates in this regime. In other words, the increase in core hole screening produces a larger shift than that arising from a decrease in the charge transfer per $\mathrm{Au}$ atom. During the late growth phase, as the NPs grow bigger and begin to merge together due to coalescence and/or Ostwald ripening, ${ }^{40}$ the charge transfer per gold atom approaches zero and, hence, the Au $4 \mathrm{f}$ binding energy increases. Although this interpretation is in line with previous calculations of the $\mathrm{Au}-\mathrm{TiO}_{2}(110)$, at first sight, it is not consistent with recent calculations that predict the formation of neutral or cationic nanoparticles. ${ }^{16}$

\section{SUMMARY}

A novel combination of STM, UV-PEEM, and $\mu$-XPS has been employed to study the interaction between the surface of $\mathrm{TiO}_{2}(110)$ and Au nanoparticles in the coverage range 0.05-1.8 MLE. The Au $4 \mathrm{f}$ photoemission binding energies of size-selected nanoparticles were measured as a monitor of the charge transfer to the nanoparticles and the screening of the core hole. Nanoparticles were fabricated using an in situ STM, using a tip shadowing method to generate a surface pattern of different coverages of Au nanoparticles. The pattern was aligned using UV-PEEM to realize $\mu$-XPS measurements of each discrete region successively. This has the advantage of allowing the measurement of different sized nanoparticles on the same substrate. $\mu$-XPS spectra were recorded in a background pressure of $10^{-6}$ mbar of $\mathrm{O}_{2}$ to minimize the effect of beam reduction of the substrate. The $\mu$-XPS data show Au $4 \mathrm{f}$ binding energies at the ultradilute limit ( $0.05 \mathrm{MLE})$ are similar to those at a higher coverage (1.8 MLE), arising from a cancellation of initial and final state effects for $\mathrm{Au}_{1}$ and $\mathrm{Au}_{3}$. As the coverage increases, the binding energy initially decreases and subsequently increases. This is consistent with charge transfer to $\mathrm{Au}_{1}$ and $\mathrm{Au}_{3}$ bound to oxygen vacancies in the ultradilute limit.

\section{ACKNOWLEDGMENTS}

This work was funded by a European Research Council grant ENERGYSURF (GT), EPSRC (Grant No. GR/R45680/01), and a Royal Society Wolfson Merit Award to GT. A.M. was also supported by SABIC UK. A.W. was supported through the EU Horizon 2020 research and innovation programme under the Marie Skłodowska-Curie Grant Agreement (GA) No. 665593 awarded to the UK Science and Technology Facilities Council.

\section{REFERENCES}

${ }^{1}$ G. Wong and J. Vohs, Surf. Sci. 498, 266-274 (2002).

${ }^{2}$ T. Minato, T. Susaki, S. Shiraki, H. S. Kato, M. Kawai, and K.-I. Aika, Surf. Sci. 566-568, 1012-1017 (2004).

${ }^{3}$ F. S. Roberts, S. L. Anderson, A. C. Reber, and S. N. Khanna, J. Phys. Chem. C 119, 6033-6046 (2015).

${ }^{4}$ J. A. Rodriguez, Catal. Today 160, 3-10 (2011).

${ }^{5}$ R. Si, J. Tao, J. Evans, J. B. Park, L. Barrio, J. C. Hanson, Y. Zhu, J. Hrbek, and J. A. Rodriguez, J. Phys. Chem. C 116, 23547-23555 (2012).

${ }^{6}$ M. Haruta, Catal. Today 36, 153-166 (1997).

${ }^{7}$ M. Valden, X. Lai, and D. W. Goodman, Science 281, 1647-1650 (1998).

${ }^{8}$ M. S. Chen and D. W. Goodman, Catal. Today 111, 22-33 (2006).

${ }^{9}$ X. Y. Liu, A. Wang, T. Zhang, and C. Y. Mou, Nano Today 8, 403-416 (2013).

${ }^{10}$ J. Grunwaldt and A. Baiker, J. Phys. Chem. B 103(6), 1002-1012 (1999).

${ }^{11}$ G. J. Hutchings, ACS Cent. Sci. 4, 1095 (2018).

${ }^{12}$ D. A. Panayotov and J. R. Morris, Surf. Sci. Rep. 71, 77-271 (2016).

${ }^{13}$ J. Radnik, C. Mohr, and P. Claus, Phys. Chem. Chem. Phys. 5, 172-177 (2003).

${ }^{14}$ Z. Jiang, W. Zhang, L. Jin, X. Yang, F. Xu, J. Zhu, and W. Huang, J. Phys. Chem. C 111, 12434-12439 (2007).

${ }^{15}$ D. Matthey, J. G. Wang, S. Wendt, J. Matthiesen, R. Schaub, E. Laegsaard, B. Hammer, and F. Besenbacher, Science 315, 1692-1696 (2007).

${ }^{16}$ S.-H. Yoo, N. Siemer, M. Todorova, D. Marx, and J. Neugebauer, J. Phys. Chem. C 123, 5495-5506 (2019).

${ }^{17}$ C. M. Yim, C. L. Pang, and G. Thornton, Phys. Rev. Lett. 104, 036806 (2010).

${ }^{18}$ U. Diebold, Appl. Surf. Sci. 48, 53-229 (2003).

${ }^{19}$ C. L. Pang, R. Lindsay, and G. Thornton, Chem. Rev. 113, 3887-3948 (2013).

${ }^{20}$ C. L. Pang, R. Lindsay, and G. Thornton, Chem. Soc. Rev. 37, 2328-2353 (2008).

${ }^{21}$ A. C. Papageorgiou, N. S. Beglitis, C. L. Pang, G. Teobaldi, G. Cabailh, Q. Chen,

A. J. Fisher, W. A. Hofer, and G. Thornton, Proc. Natl. Acad. Sci. U. S. A. 107, 2391-2396 (2010). 
${ }^{22}$ T. Minato, Y. Sainoo, Y. Kim, H. S. Kato, K.-I. Aika, M. Kawai, J. Zhao, H. Petek, T. Huang, W. He, B. Wang, Z. Wang, Y. Zhao, J. Yang, and J. G. Hou, J. Chem. Phys. 130, 124502 (2009).

${ }^{23}$ M. Setvin, C. Franchini, X. Hao, M. Schmid, A. Janotti, M. Kaltak, C. G. Van de Walle, G. Kresse, and U. Diebold, Phys. Rev. Lett. 113, 086402 (2014).

${ }^{24}$ C. M. Yim, M. B. Watkins, M. J. Wolf, C. L. Pang, K. Hermansson, and G. Thornton, Phys. Rev. Lett. 117, 116402 (2016).

${ }^{25}$ S. Chretien and H. J. Metiu, J. Chem. Phys. 127, 244708 (2007).

${ }^{26}$ X. Tong, L. Benz, S. Chretien, H. Metiu, M. T. Bowers, and S. K. Buratto, J. Phys. Chem. C 114, 3987-3990 (2010).

${ }^{27}$ S. Lee, C. Fan, T. Wu, and S. L. Anderson, J. Am. Chem. Soc. 126, 5682-5683 (2004).

${ }^{28}$ A. Mellor, D. S. Humphrey, C. M. Yim, C. L. Pang, H. Idriss, and G. Thornton, J. Phys. Chem. C 121(44), 24721-24725 (2017).

${ }^{29}$ S. C. Parker and C. T. Campbell, Top. Catal, 44, 3-13 (2007).

${ }^{30}$ A. Maeland and T. B. Flanagan, Can. J. Phys. 42(11), 2364-2366 (1964).
${ }^{31}$ A. Kolmakov and D. W. Goodman, Rev. Sci. Instrum. 74(4), 2444-2450 (2003).

${ }^{32}$ A. Wilson, R. Bernard, Y. Borensztein, B. Croset, H. Cruguel, A. Vlad, A. Coati, Y. Garreau, and G. Prevot, J. Phys. Chem. Lett. 6, 2050-2055 (2015).

${ }^{33}$ N. S. Phala and E. van Steen, Gold Bull. 40, 150-153 (2007).

${ }^{34}$ L. Gregoratti, T. O. Mentes, A. Locatelli, and M. Kiskinova, J. Electron Spectrosc. Relat. Phenom. 170, 13-18 (2009).

${ }^{35}$ A. Locatelli, T. Pabisiak, A. Pavlovska, T. O. Mentes, L. Aballe, A. Kiejna, and E. Bauer, J. Phys.: Condens. Matter 19, 082202 (2007).

${ }^{36}$ D. C. Grinter, C. Muryn, A. Sala, C. M. Yim, C. L. Pang, T. O. Mentes, A. Locatelli, and G. Thornton, J. Phys. Chem. C 120, 11037-11044 (2016).

${ }^{37}$ M. L. Knotek and P. J. Feibelman, Phys. Rev. Lett. 40, 964-967 (1978).

${ }^{38}$ D. T. Atwood, Soft X-Rays and Extreme Ultraviolet Radiation: Principle and Applications (Cambridge University Press, 1999).

${ }^{39}$ N. Spiridis, J. Haber, and J. Korecki, Vacuum 63, 99-105 (2001).

${ }^{40}$ R. Lazzari, G. Renaud, J. Jupille, and F. Leroy, Phys. Rev. B 76, 125412 (2007). 